\title{
Assembling the Digital Mathematics Library
}

\author{
David Ruddy \\ Project Euclid, Cornell University \\ dwr4@cornell.edu
}

Since discussions of a Digital Mathematics Library (DML) were first formalized, it has been recognized that such a collection would be federated, consisting of a "network of institutions." Implicit in this conception, and explicit in much of the early DML planning documents, is the assumption that this network would be organized in some manner-coordinated and held together by formally accepted policies and practices regarding collection, management, access, and preservation.

This DML, so conceived, has not come to pass. I suggest this is not because the early vision of a DML was particularly flawed, but because, for one, it was enormously more complex than we thought, and two, the approach taken was beyond our capabilities. However inevitable such an approach was, it was unrealistic given our capacity and understanding. We did not, and still do not, possess the technical understanding, the organizational capabilities, or the institutional and political willingness to implement such a grand vision in the manner proposed.

I will argue that the way forward need not abandon the larger vision but rather set aside many of the constructs upon which we assumed it needed to be realized. Chief among these is the notion of a coordinated, planned, or even sensible approach to building the DML-the idea that a central organizing network of institutions will establish, through some formal process, a plan to accomplish the goals of the DML. The future DML, if we can even call it a library, will not be "organized" (in any conventional sense of the word), at least for many years, if ever.

This is not, in my opinion, bad news or even pessimistic. It is rather a natural and expected evolution in our progress. And there has been progress, most notably in our thinking about large scale document networks. There are, further, I will argue, constructive areas of work ahead. For one, we can encourage and promote low-barrier local practices that we are increasingly confident will contribute to a large scale federated digital collection. Such local efforts include digitization methods, local data management practices, and adopting less fearful and more constructive procedures for exposing content. Second, we can engage in more exploration of how to operate in a messy information space, not with the goal of curating or exerting control over a disparate set of data, but aimed at connecting the dots. We should recognize that it is the relationships among exposed content that deserve our attention in this effort. What we may find frustrating about these relationships, their dynamic, shifting, multitudinous nature, is in fact the living nature of our future information environment and the source of its richness. 